\title{
Microfluidic-based point-of-care testing for disease diagnosis
}

\author{
Ruixuan Wen ${ }^{1}$ \\ ${ }^{1}$ Kunshan International School, 288 Qingyang Middle Rd, Kunshan, Suzhou, Jiangsu, China
}

\begin{abstract}
The early detection of diseases is important to optimize clinical treatment. To meet the increasing requirement of rapid medical diagnosis, point-of-care testing (POCT) has drawn scientists' great attention for its outstanding advantages including simple, efficient, inexpensive and instrument-independent. Recent advantages of microfluidic-based diagnosis have played an important role in the significant technological process of POCT, which can achieve rapid diagnosis and provide high precision testing results for correct clinical decision making. This review focuses on design strategies, detection targets and sensing mechanisms of recent microfluidic-based POCT for disease diagnosis. Further, we provide a perspective of the next generation of POCT devices and their future opportunities.
\end{abstract}

\section{Introduction}

Early detection of disease is necessary for it may result in optimized treatment and reducing mortality. Recently, point-of-care testing (POCT), a simple, efficient, userfriendly, inexpensive and instrument-independent portable analytic system, has been developed to achieve accurate and rapid disease diagnosis. [1-4] The diagnosis results can be quickly obtained using POCT system, which enables a more effective disease therapy and a correct use of drug. Therefore, POCT is very useful for disease prevention, diagnosis as well as the control of disease outbreak, especially in countries with low medical resources.

During last two decades, there is a significant technological progress in microfabrication technologies, which has already become an essential research area for microfluidic applications in pharmaceutics, environment and biomedical engineering research area. [5-7] In particular, biomedical microsystems have already shown an extremely positive potential for POCT application, widely employed for high-precision material synthesis, preparation of biochemical sample, and biophysical analysis. [8-10]

In this review, we focus on the microfabrication techniques, disease biomarkers and the detection strategies to demonstrate the design of microfluidic POCT systems as well as their future perspectives, which will contribute to the development of nextgeneration POCT system.

\section{Microfluidic device fabrication}

At present, various microfabrication technologies have been developed to achieve the development of microfluidic devices, which can be fabricated by various materials including glass and silicon, polymers, and paper.

\subsection{Glass and silicon}

Glass and silicon wafers were used for the first generation of microfluidic chips. Micromachining and photolithography on glass and oxidized silicon have been well developed during 90s decade, which makes them suitable for biological and biomedical applications. [5,11-13] The fabrication of this type of microfluidic devices is usually achieved in a clean room, which requires complicated equipment. Although the cost is high, glass or silicon based microfluidic devices are very reliable and precise, which can achieve on-chip reactions, capillary electrophoresis, droplet formation and solvent extraction in extreme test. Digital microfluidics is an important class of glass or silicon-based microfluidics. As shown in Fig 1A, Wan et al. has developed a digital microfluidic system using glass to detect specific pathogen through loop-mediated isothermal amplification. [14] 
A a

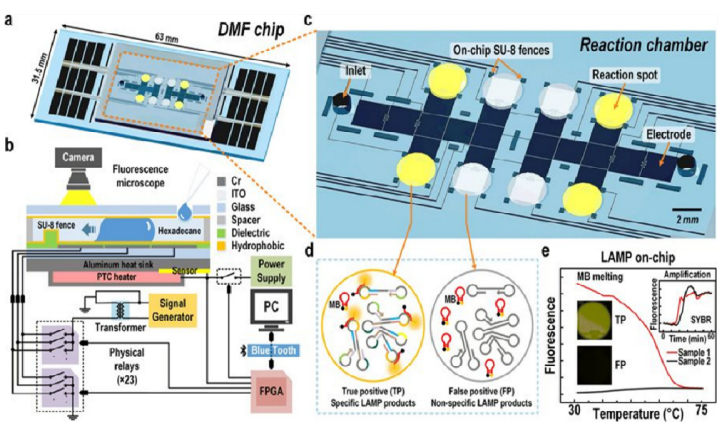

B

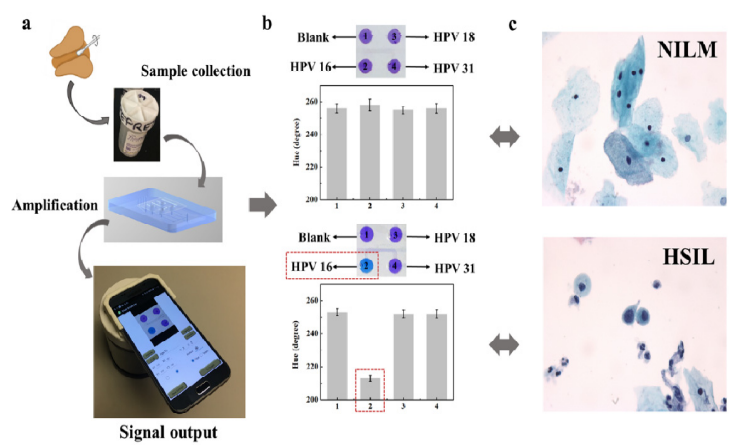

C

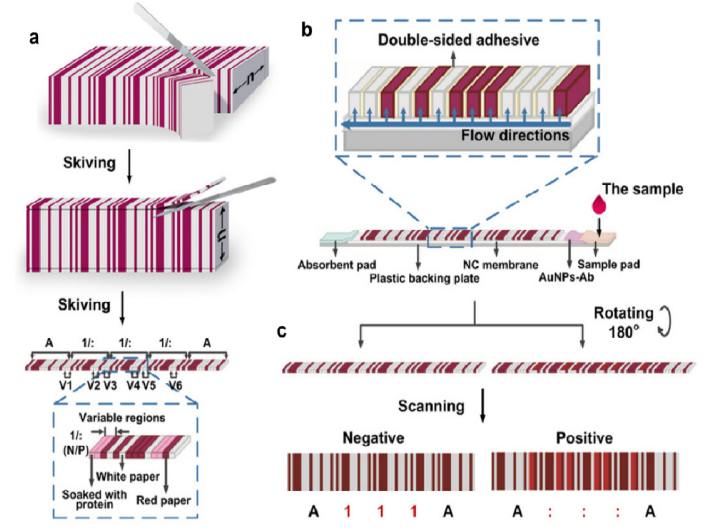

Figure 1. Microfabrication technologies for POCT. A: digital microfluidic system using glass to detect specific pathogen. B: colorimetric molecular detection method using PMMA-based microfluidic device for HPV-associated cancer screening. C: test paper for rapid and multiplexed assay.

\subsection{Polymers}

Polymeric and organic materials are most widely used to fabricate microfluidic devices for biomedical application. Compared with glass or silicon-based microfluidics, the fabrication process of polymer-based microfluidic device is based on replication. Therefore, the fabrication of microfluidic devices using polymers is faster and less expensive, which is more suitable for mass production. Additionally, polymer-based microfluidics are easy processing, low-cost, flexibility and meantime owns a wide range of chemical and mechanical properties, which is an attractive alternative to glass and silicon. [15] The most popular materials for polymer-based microfluidic systems are poly (methylmethacrylate) (PMMA) and poly(dimethylsiloxane) (PDMS). As shown in Fig. 1B, Yin et al., has developed a colorimetric molecular detection method using PMMA- based microfluidic device for HPV-associated cancer screening. [2]

\subsection{Paper}

Paper is a type of useful substrate for microfluidic device fabrication. Paper-based microfluidic device is flexible, low-cost, disposable and adaptable to large-scale manufacturing. [16] Typically, the microfluidic analytical devices using paper do not require external power because of the capillary effect of cellulose fibers in paper. Considering that cellulose fibers sometimes are partially filled in channels of closed-channel paper-based microfluidic devices, some open-channel devices have been developed using embossing, folding, engraving and embossing cellophane methods, which can achieve similar functions with conventional polymer-based microfluidic devices. As shown in Fig. 1C, Yang et al. has developed a test paper for rapid and multiplexed assay. [17]

\section{Disease biomarkers}

The specific identification of biological biomarkers is the key point for the detection of pathogens. The recognition of targets is usually based on two different ways:1) recognize the membrane specific epitopes using antibody and its alternatives; 2) recognize the specific nucleic acid.

\subsection{Antibody and its alternatives}

For the detection of pathogens, antibody-based system is one of the major analytical methods (Fig. 2A). ${ }^{[18,19]}$ Despite labor-intensiveness, this technique has proven to be very important in the high-affinity and specific detection of disease biomarkers. Whole cell detection and specific protein detection are the two most widely used antibody-based methods for disease diagnosis. However, traditional antibody-based detection technique has several drawbacks, including expensive cost, poor physical and chemical stability, and large size, which limits its application for certain disease biomarkers. To address these drawbacks, some alternatives to antibody have been developed such as molecularly imprinted polymers (MIPs) and aptamers. MIPs can be produced at low cost with high reproducibility and stability, which have been used for the detection of human rhinovirus serotype 2 and tobacco mosaic virus. [20,21] Aptamers are another recently-developed alternatives to replace antibodies. [22] Aptamers are usually isolated by the systematic evolution of ligands by exponential enrichment (SELEX) technique. [23] They are molecules of nucleic acid developed by an in vitro process, which can recognize their targets(e.g. proteins, cells, etc.) with high specificity and affinity, which can achieve better limit of detection compared with traditional antibodies. 


\subsection{DNA/RNA}

Compared with traditional antibody-based approach, DNA/RNA-based detection methods have outstanding advantages because of their capabilities for rapid, specific and sensitive detection of target nucleic acids (Fig.2B). PCR (polymerase chain reaction) is the most widely used molecular technique for DNA amplification. It has already played an important role in disease diagnosis. However, PCR process includes three temperature steps, which is not easy to develop point-ofcare devices. Therefore, isothermal amplification has drawn great attention because the reaction can be achieved at one temperature, which means that it is simpler and energy efficient. The loop-mediated isothermal amplification (LAMP), recombinase polymerase amplification (RPA), rolling circle amplification (RCA) and helicase-dependent amplification (HDA) are some established isothermal amplification methods. [24-27]

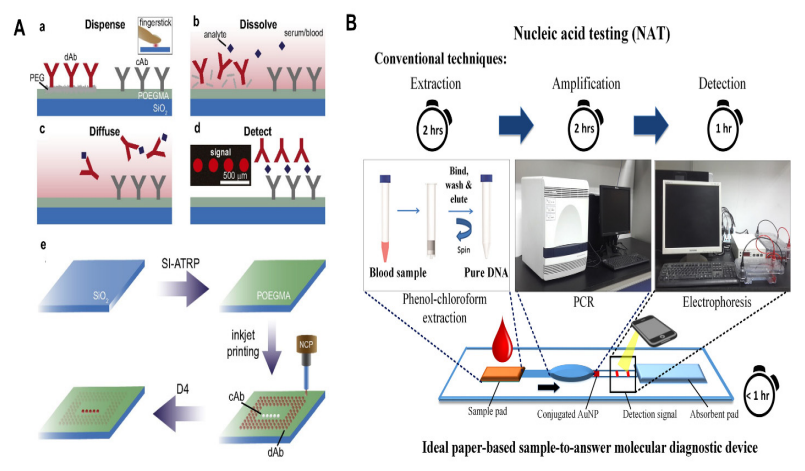

Figure. 2 The point-of-care testing for disease diagnosis based on the recognition of antibody (A) and nucleic acids (B).

\section{Detection strategies of POCT}

It is crucial to develop a low-cost, user-friendly, and efficient diagnostic system with high accuracy and sensitivity. Up to now, a variety of POCT technologies have been developed for the early disease diagnosis, which can contribute to preventing the outbreak of many pandemic diseases.

\subsection{Optical POCT devices}

Taking advantages of various optical instruments such as lenses, diffraction gratings, light sources and filters, optical based POCT devices can realize colorimetric, fluorescence, luminescence-based detection.

\subsubsection{Colorimetric POCT}

Colorimetric detection measures the absorbance variations induced by plasmon resonance phenomena or structural shifts. The colour difference can be directly recognized by naked eyes or detected by spectrophotometer. For example, a POCT device has been developed to simultaneously detect CHIKV, ZIKV, and DENV based on multiplexed RT-LAMP assay (Fig.3A). [28]

\subsubsection{Fluorescence POCT}

Fluorescence testing has been widely used in the field of chemistry, biomedicine, and biotechnology because of its outstanding advantages of high sensitivity, ease of manipulation and non-invasiveness, etc. As shown in Fig. 3B, a cost-effective and field-portable POC nucleic acid testing platform has been developed based on fluorescence-based assay. [29]

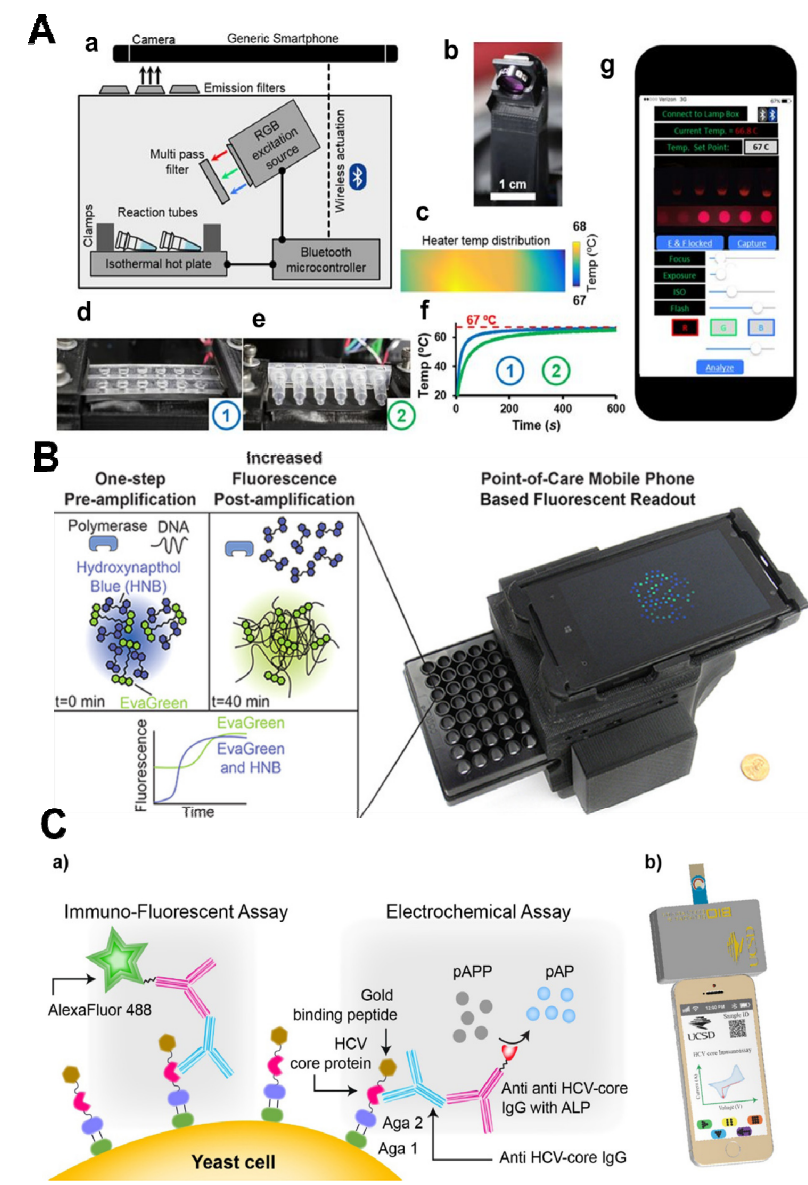

Figure. 3 Different detection strategies of POCT. A: colorimetric-based POCT; B: fluorescent-based POCT; C: electrochemical-based POCT.

\subsubsection{Luminescence POCT}

Luminescence-based POCT usually owns higher sensitivity than those based on colorimetric reading. At the same time, they can be successfully operated without light source. However, luminescence POCT is timedependent decaying of a luminescent reaction, which will lead to some limitations for consistent analysis.

\subsection{Electrochemical POCT devices}

Electrochemical POCT can provide higher reproducibility and sensitivity than optical POC diagnosis methods. As shown in Fig. 3C, Aronoff et al. has established an integrated approach to detect hepatitis 
$\mathrm{C}$ virus antibodies using electrochemical POC platform, which can also be applied for other antigens such as detect malaria. [30]

\section{Conclusion and future perspectives}

Microfluidic POCT possesses outstanding advantages over traditional diagnosis methods. For example, microfluidic POCT can reduce the consumption of clinical samples and reagents. Additionally, they are operational automatic and low-costed. Moreover, the high surface-to-volume ratio of microfluidic POCT can inhale mass transport, resulting in greater reliability and shorter trial times and greater reliability.

To achieve better detection, the reaction signal can be further enhanced by some advanced material including golden nanoparticle and fluorescence probes. Additionally, the detection signal can be transferred to smartphone-based devices for further result evaluation. The development of connected electronic devices and worldwide communication system may lead to the next generation of POC diagnosis.

The location labeling testing method can be used for the monitoring and detection of diseases with the help of smartphone-based POCT system. In addition, 3D printing technology can enhance the performance of microfluidic POCT device because of their outstanding advantages including fast and one-step fabrication, which does not require complex laboratory equipment. With the help of 3D printing and smartphone sensing technology, sensitive and low-cost detection devices can be developed. Briefly, the next generation of microfluidic POCT platform with the help of advanced materials, 3D printing technology, smartphone system, and internet clouds will have a huge potential to revolutionize the global health care system.

\section{References}

1. Wang, S. et al. Advances in addressing technical challenges of point-of-care diagnostics in resourcelimited settings. Expert review of molecular diagnostics 16, 449-459 (2016).

2. Yin K, P. V., Kadimisetty K, Ruiz C, Cooper K, You J, Liu C. Synergistically enhanced colorimetric molecular detection using smart cup: a case for instrument-free HPV-associated cancer screening. Theranostics 9, 9 (2019).

3. Luppa, P. B., Müller, C., Schlichtiger, A. \& Schlebusch, H. Point-of-care testing (POCT): Current techniques and future perspectives. $\operatorname{Tr} A C$ Trends in Analytical Chemistry 30, 887-898 (2011).

4. Vashist, S. K., Luppa, P. B., Yeo, L. Y., Ozcan, A. \& Luong, J. H. Emerging technologies for nextgeneration point-of-care testing. Trends in biotechnology 33, 692-705 (2015).

5. Zare, R. N. \& Kim, S. Microfluidic platforms for single-cell analysis. Annual review of biomedical engineering 12, 187-201 (2010).
6. Bridle, H., Miller, B. \& Desmulliez, M. P. Application of microfluidics in waterborne pathogen monitoring: A review. water research 55, 256-271 (2014).

7. Zhang, L. et al. Point-of-care-testing of nucleic acids by microfluidics. TrAC Trends in Analytical Chemistry 94, 106-116 (2017).

8. Zhang, X. et al. Smart ring: a wearable device for hand hygiene compliance monitoring at the point-ofneed. Microsystem Technologies, 1-6 (2018).

9. Urban, G. A. Micro-and nanobiosensors-state of the art and trends. Measurement science and Technology 20, 012001 (2008).

10. Gervais, L., De Rooij, N. \& Delamarche, E. Microfluidic chips for point - of - care immunodiagnostics. Advanced materials 23, H151H176 (2011).

11. Faustino, V., Catarino, S. O., Lima, R. \& Minas, G. Biomedical microfluidic devices by using low-cost fabrication techniques: A review. Journal of biomechanics 49, 2280-2292 (2016).

12. Qin, D., Xia, Y. \& Whitesides, G. M. Soft lithography for micro-and nanoscale patterning. Nature protocols 5, 491 (2010).

13. Mitra, S. K. \& Chakraborty, S. Microfluidics and nanofluidics handbook: fabrication, implementation, and applications. (CRC press, 2016).

14. Wan, L. et al. A digital microfluidic system for loopmediated isothermal amplification and sequence specific pathogen detection. Scientific reports 7, 14586 (2017).

15. Pinto, E. et al. A rapid and low-cost nonlithographic method to fabricate biomedical microdevices for blood flow analysis. Micromachines 6, 121-135 (2015).

16. Mou, L. \& Jiang, X. Materials for microfluidic immunoassays: a review. Advanced healthcare materials 6, 1601403 (2017).

17. Yang, M. et al. Skiving stacked sheets of paper into test paper for rapid and multiplexed assay. Science advances 3, eaao4862 (2017).

18. Nimse, S. B., Sonawane, M. D., Song, K.-S. \& Kim, T. Biomarker detection technologies and future directions. Analyst 141, 740-755 (2016).

19. Joh, D. Y. et al. Inkjet-printed point-of-care immunoassay on a nanoscale polymer brush enables subpicomolar detection of analytes in blood. Proceedings of the National Academy of Sciences 114, E7054-E7062 (2017).

20. BelBruno, J. J. Molecularly imprinted polymers. Chemical reviews 119, 94-119 (2018).

21. Birnbaumer, G. M. et al. Detection of viruses with molecularly imprinted polymers integrated on a microfluidic biochip using contact-less dielectric microsensors. Lab on a Chip 9, 3549-3556 (2009). 
22. Tombelli, S., Minunni, M. \& Mascini, M. Analytical applications of aptamers. Biosensors and Bioelectronics 20, 2424-2434 (2005).

23. Sefah, K., Shangguan, D., Xiong, X., O'donoghue, M. B. \& Tan, W. Development of DNA aptamers using Cell-SELEX. Nature protocols 5, 1169 (2010).

24. Mori, Y. \& Notomi, T. Loop-mediated isothermal amplification (LAMP): a rapid, accurate, and costeffective diagnostic method for infectious diseases. Journal of infection and chemotherapy 15, 62-69 (2009).

25. El Wahed, A. A. et al. A portable reverse transcription recombinase polymerase amplification assay for rapid detection of foot-and-mouth disease virus. PloS one 8, e71642 (2013).

26. James, A., Geijskes, R., Dale, J. \& Harding, R. Development of a novel rolling-circle amplification technique to detect Banana streak virus that also discriminates between integrated and episomal virus sequences. Plant disease 95, 57-62 (2011).

27. Niemz, A., Ferguson, T. M. \& Boyle, D. S. Point-ofcare nucleic acid testing for infectious diseases. Trends in biotechnology 29, 240-250 (2011).

28. Priye, A. et al. A smartphone-based diagnostic platform for rapid detection of Zika, chikungunya, and dengue viruses. Scientific reports 7, 44778 (2017).

29. Kong, J. E. et al. Highly stable and sensitive nucleic acid amplification and cell-phone-based readout. ACS nano 11, 2934-2943 (2017).

30. Aronoff-Spencer, E. et al. Detection of Hepatitis C core antibody by dual-affinity yeast chimera and smartphone-based electrochemical sensing. Biosensors and Bioelectronics 86, 690-696 (2016). 